\title{
Switchable diurnal radiative cooling by doped $\mathrm{VO}_{2}$
}

\author{
Minkyung $\mathrm{Kim}^{1 \dagger}$, Dasol Lee ${ }^{1 \dagger}$, Younghwan Yang ${ }^{1}$ and Junsuk Rho ${ }^{1,2 *}$
}

This paper presents design and simulation of a switchable radiative cooler that exploits phase transition in vanadium dioxide to turn on and off in response to temperature. The cooler consists of an emitter and a solar reflector separated by a spacer. The emitter and the reflector play a role of emitting energy in mid-infrared and blocking incoming solar energy in ultraviolet to near-infrared regime, respectively. Because of the phase transition of doped vanadium dioxide at room temperature, the emitter radiates its thermal energy only when the temperature is above the phase transition temperature. The feasibility of cooling is simulated using real outdoor conditions. We confirme that the switchable cooler can keep a desired temperature, despite change in environmental conditions.

Keywords: phase change material; photonic crystal; passive thermoregulation; switchable radiative cooling; Fabry-Pérot resonance

Kim MK, Lee DS, Yang YH, Rho JS. Switchable diurnal radiative cooling by doped VO 2 . Opto-Electron Adv 4, 200006 (2021).

\section{Introduction}

Every object with finite temperature emits heat by thermal radiation. If an object radiates more energy than it absorbs from its surroundings, it loses energy and cools down. This phenomenon occurs generally at night when the object is not subject to solar irradiation, and forms a basis of nocturnal radiative cooling ${ }^{1-3}$. However, it has been recently suggested that a material that reflects solar energy but radiates in an atmospheric window (wavelengths $8 \mu \mathrm{m}<\lambda<13 \mu \mathrm{m}$ ) can lose energy during the day ${ }^{4}$. This observation has stimulated studies on diurnal radiative cooling, in which an object can cool down even under solar illumination without consuming external energy ${ }^{5-9}$. Therefore, diurnal radiative cooling has been evaluated as an alternative cooling technology for future.

For efficient radiative cooling, two conditions should be satisfied. The absorptivity of the object should be near zero in the ultraviolet (UV) to near-infrared (NIR) range, and should radiate its energy with near-unity efficiency in the wavelengths at which electromagnetic radiation can be transmitted through the atmosphere (the "atmospheric window"). To meet those conditions, structured materials such as photonic crystals ${ }^{10}$, micro/nanoporous structures $^{11-17}$, multilayer ${ }^{18,19}$, and mixtures of dielectric particles $^{20-23}$ have been used for their ability to manipulate optical responses. However, the spectra of such media cannot be changed after fabrication. Therefore, many efforts have been devoted to find optical materials and structures that have switchable thermal characteristics.

An interesting example is the use of vanadium dioxide $\left(\mathrm{VO}_{2}\right)$. It is a phase change material that has an insulating phase at temperature $T$ below the critical temperature $T_{\mathcal{c}}$ and metallic phase at $T>T_{\mathcal{c}}$. The phase

1Department of Mechanical Engineering, Pohang University of Science and Technology (POSTECH), Pohang 37673, Republic of Korea; 2Department of Chemical Engineering, Pohang University of Science and Technology (POSTECH), Pohang 37673, Republic of Korea.

†These authors contributed equally to this work.

*Correspondence: JS Rho, E-mail: jsrho@postech.ac.kr

Received: 28 February 2020; Accepted: 22 June 2020; Published: 20 May 2021

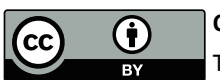

Open Access This article is licensed under a Creative Commons Attribution 4.0 International License.

To view a copy of this license, visit http://creativecommons.org/licenses/by/4.0/.

(C) The Author(s) 2021. Published by Institute of Optics and Electronics, Chinese Academy of Sciences. 
transition of $\mathrm{VO}_{2}$ has been used to develop active emitters and absorbers ${ }^{24-26}$, switchable polarization rotators ${ }^{27}$, waveguides ${ }^{28}$ and nanoantennas ${ }^{29} . T_{\mathrm{c}}$ of pure $\mathrm{VO}_{2}$ is 68 ${ }^{\circ} \mathrm{C}$, but can be set to around room temperature by doping molybdenum, tungsten and/or strontium ${ }^{30-37}$ and by growing $\mathrm{VO}_{2}$ thin film on $\mathrm{TiO}_{2}$ substrates ${ }^{38,39}$. This relatively low $T_{\mathrm{c}}$ of $\mathrm{VO}_{2}$ encouraged the development of an active radiative cooler ${ }^{40}$ based on the transmissive filter placed on a switchable emitter and a photonic-based thermostat ${ }^{41}$ consisting of repeated layers of $\mathrm{VO}_{2}$ grown on $\mathrm{TiO}_{2}$ film and zinc selenide. Such active thermal devices radiate only at $T>T_{\mathrm{c}}$ and thus lose their thermal energies as temperature increases while maintaining temperature similar to ambient as temperature decreases. Here, we develop a switchable radiative cooler by combining two parts: an emitter that uses doped $\mathrm{VO}_{2}$, and a solar reflector that has optimized one-dimensional multi-stacked photonic crystals. The emitter part is designed to selectively radiate in the atmospheric window whereas the solar reflector blocks solar irradiance in UV to NIR regime. Thus, the cooler exhibits positive cooling power at $T>T_{\mathrm{c}}$ and negative cooling power at $T<T_{\mathrm{c}}$. Therefore, it maintains a moderate temperature that is resilient to environmental changes. Simulation using a diurnal cycle of outdoor temperature and solar radiation proved that the radiative cooler is capable of switchable

a
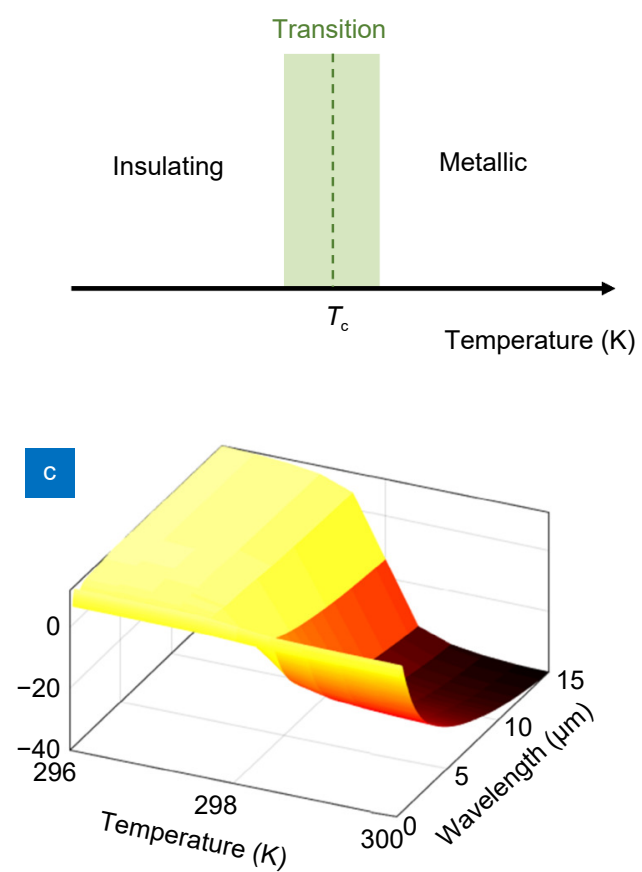

diurnal radiative cooling. In comparison to other static radiative cooler, the switchable radiative cooler exhibits minimal variation of temperature, and is therefore useful in various applications including air conditioning and heating.

\section{Results and discussion}

\section{Material properties and design}

The optical behavior of $\mathrm{VO}_{2}$ can be described as Fig. 1(a). In the infrared range, $\mathrm{VO}_{2}$ responses as a metal well above $T_{\mathrm{c}}$ and as an insulator well below $T_{\mathrm{c}}$. To avoid an unphysical result, we assume that the permittivity of $\mathrm{VO}_{2}$ changes continuously and smoothly in a narrow transition range $\left[T_{\mathrm{c}}-\Delta T, T_{\mathrm{c}}+\Delta T\right]$. Thus, the permittivity of $\mathrm{VO}_{2}$ is modelled individually in three distinct regimes: insulating, transition and metallic regime (Fig. 1(a)). For insulating and metallic regime, we use permittivities of doped $\mathrm{VO}_{2}$ presented in ref. ${ }^{40}$. In the transition regime, we assume that permittivity changes as an arctan function (Fig. 1(b)):

$\mathcal{E}_{\text {transition }}=\arctan \left(\frac{T-T_{\mathrm{c}}}{\Delta T} \times 10\right) \times \frac{\varepsilon_{\mathrm{m}}-\varepsilon_{\mathrm{i}}}{2 \arctan 10}+\frac{\varepsilon_{\mathrm{m}}+\varepsilon_{\mathrm{i}}}{2}$,

where $\varepsilon_{\mathrm{m}}$ and $\varepsilon_{\mathrm{i}}$ are permittivity in the metallic and insulating phase, respectively. Throughout this paper, we

\section{b}
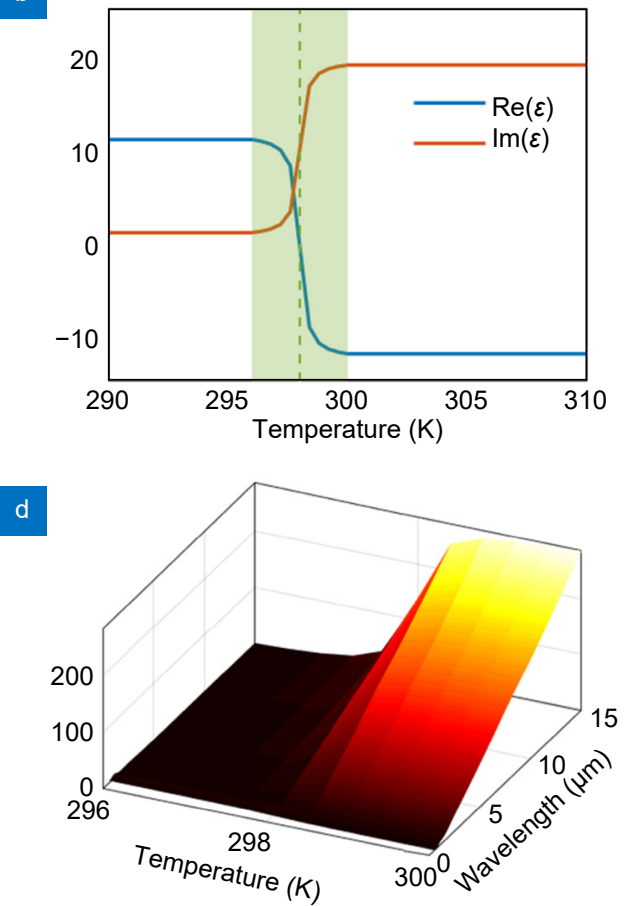

Fig. 1 | Temperature-dependent material responses of $\mathbf{V O}_{2}$. (a) Schematic of temperature-dependent phase. (b) Permittivity at $2 \mu \mathrm{m}$ when $T_{\mathrm{C}}=298 \mathrm{~K}$. Shaded area represents transition regime. (c) Real and (d) imaginary part of permittivity in transition regime. 
set $T_{\mathrm{c}}=298 \mathrm{~K}$ and $\Delta T=2 \mathrm{~K}$. In the transition regime, both the real and imaginary parts of $\varepsilon_{\text {transition }}$ change smoothly (Figs. 1(c) and 1(d)).

To minimize the absorption of solar irradiance and maximize thermal energy radiated through the atmospheric window in a switchable way, we combine an emitter and a solar reflector separated by a spacer (Fig. 2) on a $\mathrm{SiO}_{2}$ substrate. The emitter part is composed of stacked layers of silver $(200 \mathrm{~nm})$, silicon $(700 \mathrm{~nm})$ and $\mathrm{VO}_{2}(10 \mathrm{~nm})$ from bottom to top. A $300 \mathrm{~nm}$-thick spacer made of poly(methyl methacrylate) (PMMA) is deposited on the emitter. The top of the spacer is the solar reflector consisting of three stacked photonic crystals. Each photonic crystal $\left(\mathrm{PC}_{i}\right)$ is a distributed Bragg reflector (DBR) that is designed to suppress absorption at a target wavelength $\lambda_{i}$ where $\lambda_{1}=0.52 \mu \mathrm{m}, \lambda_{2}=0.76 \mu \mathrm{m}$ and $\lambda_{3}=$ $1.18 \mu \mathrm{m}$. Thickness of each layer is set as $\lambda_{i} / 4 n$ following the design rule of DBRs ${ }^{42}$.

\section{Optical responses}

The transfer-matrix method was used to calculate the absorptivity and reflectivity spectra ${ }^{43}$. We examine absorptivity and reflectivity of the emitter part and solar reflector part individually. The emitter works as a metalinsulator-metal structure at $T>T_{\mathrm{c}}$. The Fabry-Pérot res- onance due to the cavity results in high absorptivity near the atmospheric window (Fig. 3(a)). In contrast, the emitter behaves as an insulator deposited on a metal and mostly reflects at $T<T_{\mathrm{c}}$ (Fig. 3(b)). Therefore, the emitter performs the switching of radiation in the atmospheric window. Meanwhile, the solar reflector reflects solar irradiance in the UV to NIR range (Fig. 3(c)). The high reflectivity of the solar reflector originates from the optimization of photonic crystal structures. The average absorptivity of the solar reflector at $\lambda<2 \mu \mathrm{m}$ is $5.9 \%$. The combined structure of emitter separated by a spacer from the solar reflector has low absorption in UV to NIR

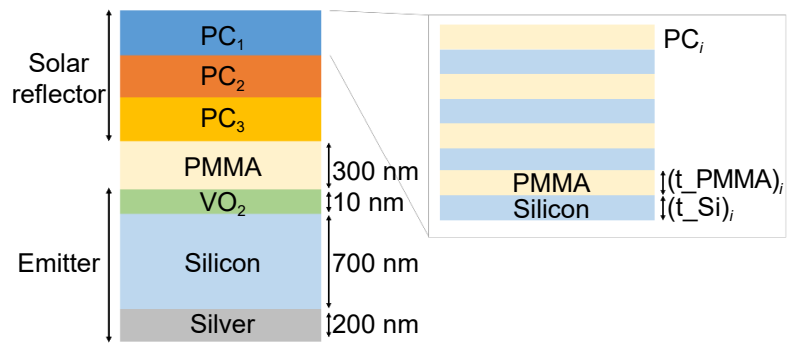

Fig. 2 | Design of the switchable radiative cooler. Emitter part consists of stacked layers of silver, silicon and $\mathrm{VO}_{2}$. Solar reflector part consists of three photonic crystals that have 4 pairs of PMMA and silicon. $\mathrm{PC}_{i}$ is designed to suppress absorption at $\lambda_{i}$ where $\lambda_{1}=$ $0.52 \mu \mathrm{m}, \lambda_{2}=0.76 \mu \mathrm{m}$ and $\lambda_{3}=1.18 \mu \mathrm{m}$. Thickness of each layer is $\lambda_{i} / 4 n$.
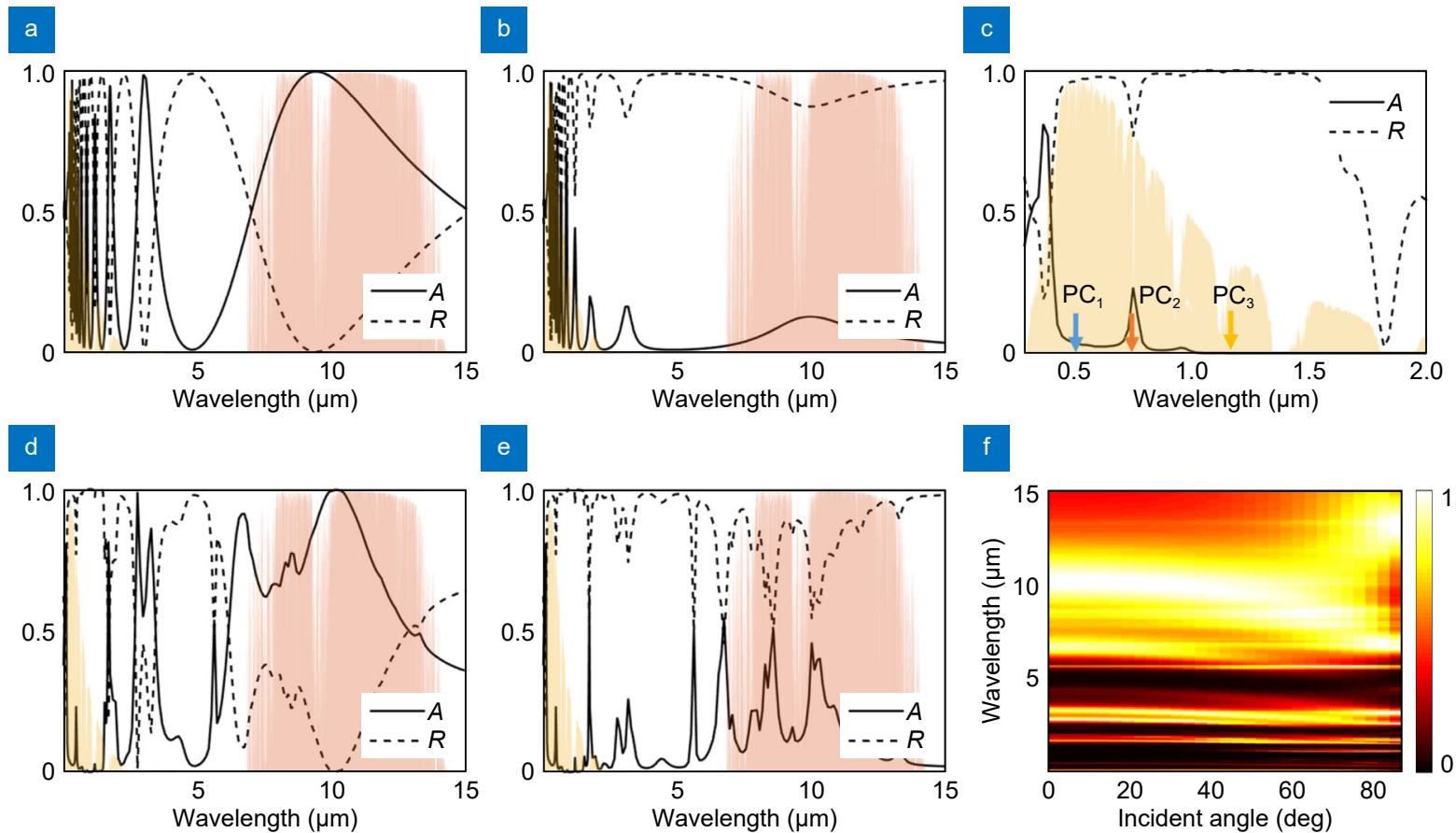

Fig. 3 | Absorptivity and reflectivity of the switchable radiative cooler. (a, b) Absorptivity and reflectivity of the emitter part when $\mathrm{VO}_{2}$ is in (a) metallic and (b) insulating state. (c) Absorptivity and reflectivity of the solar reflector part. Three arrows represent the target wavelengths of three photonic crystals. (d, e) Absorptivity and reflectivity of the switchable radiative cooler when $\mathrm{VO}_{2}$ is in (d) metallic and (e) insulating state. Incident angle is zero. (f) Absorptivity of the switchable radiative cooler when $\mathrm{VO}_{2}$ is metallic. 
and switchable absorption in the atmospheric window simultaneously. Absorptivity in UV to NIR is low in both metallic and insulating phases; the combined structure absorbs $7.6 \%$ in average. In contrast, absorptivity in the atmospheric window are remarkably distinct in two phases. The absorptivity is high in the metallic phase, but low in the insulating phase (Figs. 3(d) and 3(e)). Average absorptivity is $78 \%$ in the metallic phase and $18 \%$ in the insulating phase. At oblique incidence, the absorptivity remains high in the atmospheric window (Fig. 3(f)).

\section{Calculation of cooling flux}

To evaluate the cooling flux, we consider blackbody radiation, solar irradiance, thermal exchange with atmosphere, and two other heat exchange channels:

$$
\begin{aligned}
P(T)= & P_{\text {rad }}(T)-P_{\text {sun }} \\
& -P_{\text {atm }}\left(T_{\text {amb }}\right)-P_{\mathrm{cc}}\left(T, T_{\mathrm{amb}}\right),
\end{aligned}
$$

where $T$ is the temperature of the switchable radiative cooler, $T_{\mathrm{amb}}$ is ambient temperature, and $P_{\mathrm{rad}}(T)$ corresponds to radiation flux emitted by the cooler:

$$
P_{\text {rad }}(T)=\iint_{0}^{\infty} I_{\mathrm{BB}}(T, \lambda) E(T, \lambda, \theta) \mathrm{d} \lambda \cos \theta \mathrm{d} \Omega,
$$

where $\lambda$ is the wavelength, $\theta$ is the polar angle, and $\int \mathrm{d} \Omega=2 \pi \int_{0}^{\pi / 2} \sin \theta \mathrm{d} \theta$ is the angular integral over a hemisphere. $E$ is the emissivity of the cooler and is equal to the absorptivity according to Kirchhoff's law ${ }^{44}$. Since $E$ of $\mathrm{VO}_{2}$ depends on its temperature, we use $E(T, \lambda, \theta)$ instead of $E(\lambda, \theta)$.

$$
I_{\mathrm{BB}}(T, \lambda)=\frac{2 \mathrm{hc}^{2}}{\lambda^{5}} \frac{1}{\mathrm{e}^{\mathrm{hc} /\left(\lambda k_{\mathrm{B}} T\right)}-1},
$$

is the spectral radiance density of a blackbody at temperature $T$, where $h$ is Planck constant, $\mathrm{c}$ is the speed of light in free space, and $k_{\mathrm{B}}$ is the Boltzmann constant. The second term represents absorbed thermal flux due to solar irradiance:

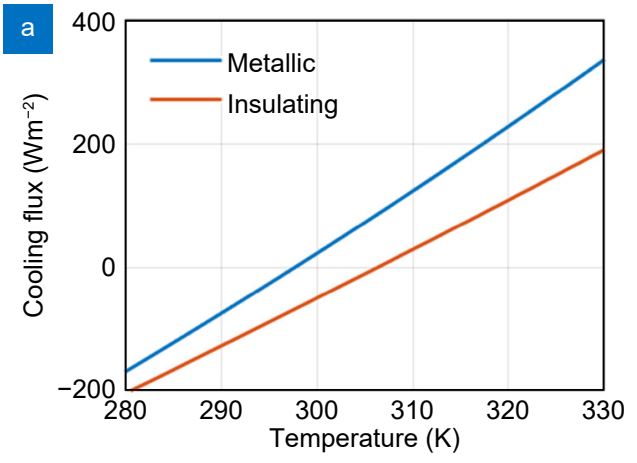

$$
P_{\text {sun }}=\int_{0}^{\infty} E(T, \lambda) I_{\mathrm{AM} 1.5}(\lambda) \mathrm{d} \lambda,
$$

where $I_{\mathrm{AM} 1.5}$ is the AM1.5 spectrum of the solar illumination. Thermal flux absorbed by the atmospheric heat exchange is

$$
\begin{aligned}
P_{\mathrm{atm}}\left(T_{\mathrm{amb}}\right)= & \iint_{0}^{\infty} I_{\mathrm{BB}}\left(T_{\mathrm{amb}}, \lambda\right) E(T, \lambda, \theta) \\
& \cdot E_{\mathrm{atm}}(\lambda, \theta) \mathrm{d} \lambda \cos \theta \mathrm{d} \Omega,
\end{aligned}
$$

where $E_{\text {atm }}=1-t(\lambda)^{1 / \cos \theta}$ is the emissivity of the atmosphere where $t(\lambda)$ is the transmittance of the atmosphere in the zenith direction ${ }^{1,45}$. The last term is associated with conduction and convection given as

$$
P_{\mathrm{cc}}\left(T, T_{\mathrm{amb}}\right)=h_{\mathrm{cc}}\left(T_{\mathrm{amb}}-T\right),
$$

where $h_{\mathrm{cc}}$ is the heat transfer coefficient due to conduction and convection. We use $h_{\mathrm{cc}}=6.9 \mathrm{Wm}^{-2} \mathrm{~K}^{-1}$ throughout the calculations.

The cooling flux $P(T)$ under normal incidence of solar energy is calculated using Eq. (2) to (7). For simplification, we first treat $E$ as a temperature-independent value. We calculate the cooling flux of the cooler by assuming that $\mathrm{VO}_{2}$ is either metallic or insulating regardless of temperature (Fig. 4(a)). When $\mathrm{VO}_{2}$ is assumed to be metallic, it has positive cooling flux above $T=297.8 \mathrm{~K}$ and negative cooling flux below it. As expected, the cooling flux when $\mathrm{VO}_{2}$ is insulating is lower than that when it is metallic. The cooling flux is zero at $T=306 \mathrm{~K}$ for the insulating case.

In reality, $E$ of $\mathrm{VO}_{2}$ varies with $T$ and thus, $E$ should be treated as a function of temperature. Cooling flux is expected to be equal to that of metallic case at $T>T_{\mathrm{c}}+\Delta T$ and to that of insulating case at $T<T_{\mathrm{c}}-\Delta T$. In the vicinity of $T_{\mathfrak{c}}$, it is assumed to change continuously and smoothly. We use Eq. (1) to calculate emissivity of the radiative cooler, then obtain the cooling flux in the transition regime when $T_{\mathrm{amb}}=303 \mathrm{~K}$ (Fig. 4(b)). The cooling flux varies by amount of more than $100 \mathrm{Wm}^{-2}$

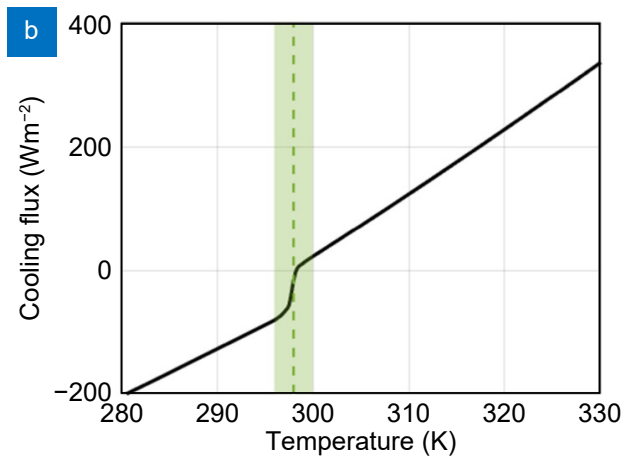

Fig. 4 | Cooling flux of the radiative cooler under normal incidence of solar energy when $T_{\mathrm{amb}}=\mathbf{3 0 3} \mathrm{K}$. (a, b) Cooling flux when permittivity of $\mathrm{VO}_{2}$ is assumed to be (a) static and (b) dynamic. Shaded area represents the transition regime. 
across the transition regime. In the atmospheric window, the cooler radiates approximately $P_{\text {rad }}=179 \mathrm{Wm}^{-2}$ when the cooling is turned on $\left(T=T_{\mathrm{c}}+\Delta T\right)$ and $P_{\mathrm{rad}}=47$ $\mathrm{Wm}^{-2}$ when the cooling is turned off $\left(T=T_{\mathrm{c}}-\Delta T\right)$. The switchable radiative cooler is in thermal equilibrium at $T=298.3 \mathrm{~K}$, which corresponds to room temperature.

\section{Numerical observation of cooling in time}

To confirm the cooling visually, we calculate how temperature of the switchable radiative cooler changes over time. Temperature variation obeys the thermal balance equation:

$$
C \frac{\mathrm{d} T}{\mathrm{~d} t}=A P\left(T, T_{\mathrm{amb}}\right),
$$

where $A$ is the area of the cooling surface, and $t$ is time. $C$ is the heat capacitance, which can be obtained by summing the heat capacitances of all layers as

$$
C=A \sum_{j} c_{j} \rho_{j} t_{j}+C_{0}
$$

where $c_{j}, \rho_{j}$ and $t_{j}$ is the specific heat, density and thickness of $j$-th layer, respectively. The summation applies to layers shown in Fig. 2 and a substrate of $500 \mu$ m-thick silicon dioxide $\left(\mathrm{SiO}_{2}\right)$. The specific heat and density (Table 1) are obtained from ref. ${ }^{46,47}$ for PMMA, ref. ${ }^{48,49}$ for silicon, ref. ${ }^{48,50}$ for silver, ref. ${ }^{48,51}$ for $\mathrm{SiO}_{2}$, and ref. ${ }^{52,53}$ for $\mathrm{VO}_{2}$. Strictly, specific heat of $\mathrm{VO}_{2}$ depends on its temperature, but we use the specific heat at room temperature because the variation range is small $\left(<3 \mathrm{Jg}^{-1} \mathrm{~K}^{-1}\right)$. To test the cooling ability, we additionally include $C_{0}=$ $1.325 \times 10^{5} \mathrm{JK}^{-1}$ as the heat capacitance of an object to be cooled; it corresponds to a block of $\mathrm{SiO}_{2}$ of unit area and $0.05 \mathrm{~m}$ thickness.

Table 1 | Specific heat and density of materials.

\begin{tabular}{c|c|c|c|c|c}
\hline & PMMA & Silicon & Silver & $\mathrm{SiO}_{2}$ & $\mathrm{VO}_{2}$ \\
\hline$c(\mathrm{~J} / \mathrm{gK})$ & 1.47 & 0.71 & 0.23 & 1.00 & 0.24 \\
\hline$\rho\left(\mathrm{g} / \mathrm{cm}^{-3}\right)$ & 1.18 & 2.33 & 10.50 & 2.65 & 4.57 \\
\hline
\end{tabular}

We simulate temperature variation in time under $T_{\mathrm{amb}}=303 \mathrm{~K}$ by using Eq. (2) and Eq. (9). For various initial temperature ranging from $280 \mathrm{~K}$ to $320 \mathrm{~K}$, temperature converges to $298.3 \mathrm{~K}$ (Fig. 5(a)). It shows that the cooler is turned on at $T>298.3 \mathrm{~K}$ but turned off at $T<$ 298.3 K. The switchable cooling is also confirmed by cooling flux which is positive at $T>298.3 \mathrm{~K}$ and negative at $T<298.3 \mathrm{~K}$ (Fig. 5(b)). The thermal equilibrium temperature can be tuned by designing the solar reflector

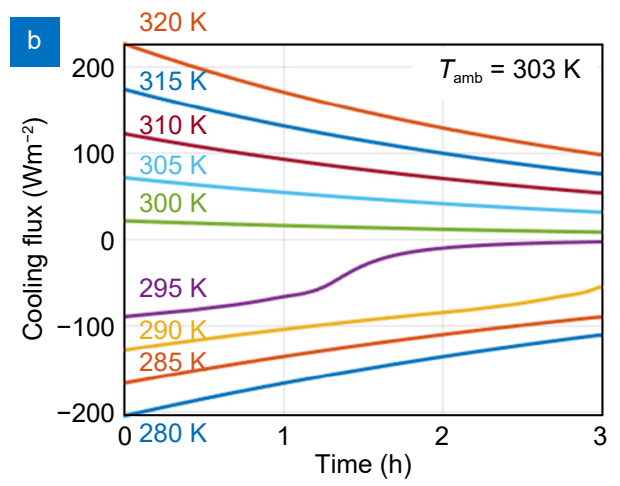

$\times 10^{3}$
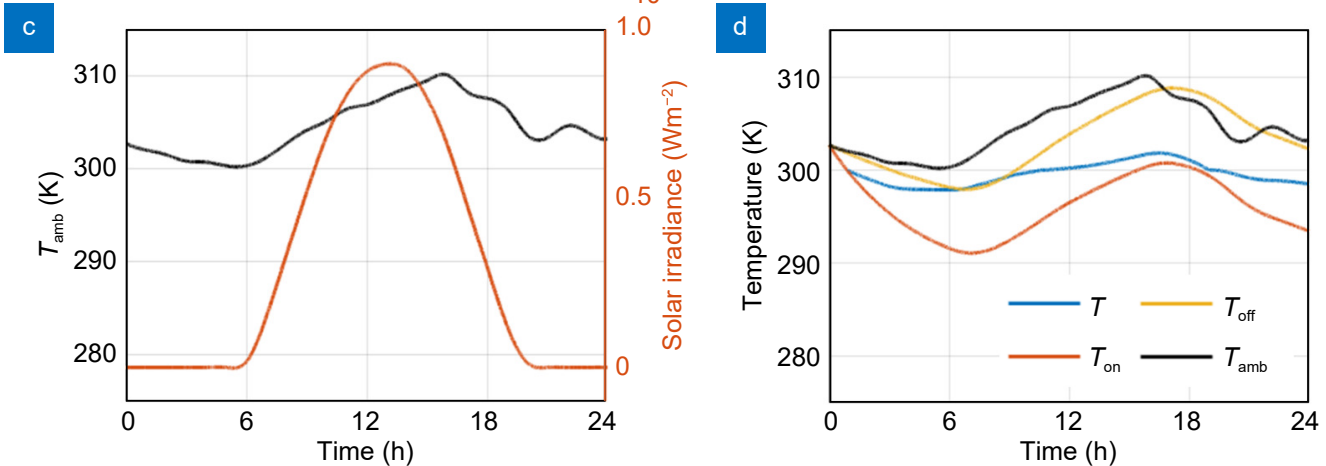

Fig. 5 | Temperature variation in time. (a) Temperature and (b) cooling flux in time for initial temperature of $280 \mathrm{~K}$ to $320 \mathrm{~K}$ with $5 \mathrm{~K}$ step. Temperature indicate the initial temperature of the cooler. $T_{\mathrm{amb}}=303 \mathrm{~K}$. (c, d) A cycle of temperature of a day. (c) $T_{\text {amb }}$ and solar irradiance of July 15, 2018 in Pohang, Korea. (d) Temperature of switchable radiative cooler (blue) and the static radiative cooler when radiative cooling is assumed to be turned on (orange) and off (yellow). $T_{\mathrm{amb}}$ is shown as a reference (black). Initial temperature of the cooler is set equal to the initial $T_{\mathrm{amb}}$. 
part. Therefore, the switchable radiative cooler provides a pathway to constantly control the temperature at a desired value.

The practicality of the switchable radiative cooler is assessed by simulating a cycle of temperature during a day. To emulate the outdoor condition, we use ambient temperature and solar irradiance $I$ measured on July 15 , 2018 in Pohang, Korea by the Korea Meteorological Administration $^{54}$ (Fig. 5(c)). Initial temperature of the switchable radiative cooler is set equal to that of ambient. During the whole day, the cooler has $T<T_{\mathrm{amb}}$, with the maximum decrement of $8.5 \mathrm{~K}$ (Fig. 5(d)). For comparison, we also plot the temperature of the radiative cooler when the cooling is turned on and off at all temperature. In all cases, temperature gets lower before 6 am when the sun rises. When the cooling is turned off, temperature increases as time passes until it reaches the equilibrium and then decreases again after the sunset. On the other hand, when the cooling is turned on, temperature decreases by approximately $10 \mathrm{~K}$ as a result of high cooling power. Temperature variation during a day exceeds $10 \mathrm{~K}$ in both cases. In contrast, the switchable radiative cooler shows a minimal change of temperature, resilient to the surrounding environment.

\section{Conclusions}

In conclusion, we present a switchable radiative cooler made of doped vanadium dioxide. The cooler emits energy in the atmospheric window only above room temperature as a result of Fabry-Pérot resonance. Thus, the cooling is conditionally turned on and off depending on the temperature. We demonstrate the switchable radiative cooling by calculating cooling flux for various temperature ranges. The switchable cooling is further supported by simulating a cycle of temperature for a day using the measured temperature and solar irradiance data. We confirm that the cooler can maintain its temperature robustly under natural weather conditions. To further improve the cooling effect of the switchable diurnal radiative cooler, the design can be optimized to have unity (zero) reflectivity in the UV to NIR range and emissivity in the atmospheric window at $T>T_{\mathrm{c}}$ (at $T<T_{\mathrm{c}}$ ) or to have the highest cooling flux by various optimization methods and machine learning ${ }^{55-57}$. The switchable radiative cooler will facilitate self-adaptive control of thermal energy and can be implemented in a variety of applications such as passive cooling in environmentally-benign buildings and vehicles.

\section{References}

1. Granqvist CG, Hjortsberg A. Radiative cooling to low temperatures: general considerations and application to selectively emitting SiO films. J Appl Phys 52, 4205-4220 (1981).

2. Gentle AR, Smith GB. Radiative heat pumping from the Earth using surface phonon resonant nanoparticles. Nano Lett 10, 373-379 (2010).

3. Catalanotti S, Cuomo V, Piro G, Ruggi D, Silvestrini V et al. The radiative cooling of selective surfaces. Sol Energy 17, 83-89 (1975).

4. Raman AP, Anoma MA, Zhu LX, Rephaeli E, Fan SH. Passive radiative cooling below ambient air temperature under direct sunlight. Nature 515, 540-544 (2014).

5. Huang $X$, Li N, Wang JF, Liu DF, Xu ZJ et al. Single nanoporous $\mathrm{MgHPO}_{4} \cdot 1.2 \mathrm{H}_{2} \mathrm{O}$ for daytime radiative cooling. ACS Appl Mater Interfaces 12, 2252-2258 (2020).

6. Ko B, Lee D, Badloe T, Rho J. Metamaterial-based radiative cooling: towards energy-free all-day cooling. Energies 12, 1-14 (2018).

7. Li T, Zhai Y, He SM, Gan WT, Wei ZY et al. A radiative cooling structural material. Science 364, 760-763 (2019).

8. Hsu PC, Song AY, Catrysse PB, Liu C, Peng YC et al. Radiative human body cooling by nanoporous polyethylene textile. Science 353, 1019-1023 (2016).

9. Li W, Fan $\mathrm{SH}$. Nanophotonic control of thermal radiation for energy applications[Invited]. Opt Express 26, 15995-16021 (2018).

10. Kou JL, Jurado Z, Chen Z, Fan SH, Minnich AJ. Daytime radiative cooling using near-black infrared emitters. ACS Photonics 4, 626-630 (2017).

11. Peng YC, Chen J, Song AY, Catrysse PB, Hsu PC et al. Nanoporous polyethylene microfibres for large-scale radiative cooling fabric. Nat Sustain 1, 105-112 (2018).

12. Mandal J, Fu YK, Overvig AC, Jia MX, Sun KR et al. Hierarchically porous polymer coatings for highly efficient passive daytime radiative cooling. Science 362, 315-319 (2018).

13. Cai LL, Song AY, Wu PL, Hsu PC, Peng YC et al. Warming up human body by nanoporous metallized polyethylene textile. Nat Commun 8, 496 (2017).

14. Yang AK, Cai LL, Zhang RF, Wang JY, Hsu PC et al. Thermal management in nanofiber-based face mask. Nano Lett 17, 3506-3510 (2017).

15. Lee D, Go M, Son S, Kim M, Badloe T et al. Sub-ambient daytime radiative cooling by silica-coated porous anodic aluminum oxide. Nano Energy 79, 105426 (2021).

16. Heo SY, Lee GJ, Kim DH, Kim YJ, Ishii S et al. A Janus emitter for passive heat release from enclosures. Sci Adv 6, 36, eabb1906 (2020).

17. Lee GJ, Kim DH, Heo SY, Song YM. Spectrally and spatially selective emitters using polymer hybrid spoof plasmonics. ACS Appl Mater Interfaces 12, 47, 53206-53214 (2020).

18. Kim M, Lee D, Son S, Yang Y, Lee H et al. Visibly transparent radiative cooler under direct sunlight. Adv Opt Mater , 2002226 (2021).

19. Chae D, Kim M, Jung PH, Son S, Seo J et al. Spectrally selective inorganic-based multilayer emitter for daytime radiative cooling. ACS Appl Mater Interfaces 12, 7, 8073-8081 (2020).

20. Atiganyanun S, Plumley JB, Han SJ, Hsu K, Cytrynbaum J et al. Effective radiative cooling by paint-format microspherebased photonic random media. ACS Photonics 5, 1181-1187 (2018).

21. Bao H, Yan C, Wang BX, Fang X, Zhao CY et al. Double-layer 
nanoparticle-based coatings for efficient terrestrial radiative cooling. Sol Energ Mater Sol C 168, 78-84 (2017).

22. Huang ZF, Ruan XL. Nanoparticle embedded double-layer coating for daytime radiative cooling. Int $J$ Heat Mass Transf 104, 890-896 (2017).

23. Zhai Y, Ma YG, David SN, Zhao DL, Lou RN et al. Scalablemanufactured randomized glass-polymer hybrid metamaterial for daytime radiative cooling. Science 355, 1062-1066 (2017).

24. Nishikawa K, Yatsugi K, Kishida Y, Ito K. Temperature-selective emitter. Appl Phys Lett 114, 211104 (2019).

25. Chen MK, Morsy AM, Povinelli ML. Design of $\mathrm{VO}_{2}$-coated silicon microspheres for thermally-regulating paint. Opt Express 27, 21787-21793 (2019).

26. Long LS, Taylor S, Ying XY, Wang LP. Thermally-switchable spectrally-selective infrared metamaterial absorber/emitter by tuning magnetic polariton with a phase-change $\mathrm{VO}_{2}$ layer. $\mathrm{Ma}$ ter Today Energy 13, 214-220 (2019).

27. Jia ZY, Shu FZ, Gao YJ, Cheng F, Peng RW et al. Dynamically switching the polarization state of light based on the phase transition of vanadium dioxide. Phys Rev Appl 9, 034009 (2018).

28. Markov P, Marvel RE, Conley HJ, Miller KJ, Haglund Jr RF et al. Optically monitored electrical switching in $\mathrm{VO}_{2}$. ACS Photonics 2, 1175-1182 (2015).

29. Shu FZ, Zhang LH, Wang JN, Peng RW, Fan RH et al. Dynamically tunable bowtie nanoantennas based on the phase transition of vanadium dioxide. Opt Lett 44, 2752-2755 (2019).

30. Dietrich MK, Kuhl F, Polity A, Klar PJ. Optimizing thermochromic $\mathrm{VO}_{2}$ by co-doping with $\mathrm{W}$ and $\mathrm{Sr}$ for smart window applications. Appl Phys Lett 110, 141907 (2017).

31. Burkhardt W, Christmann T, Franke S, Kriegseis W, Meister D et al. Tungsten and fluorine co-doping of $\mathrm{VO}_{2}$ films. Thin Solid Films 402, 226-231 (2002).

32. Jorgenson GV, Lee JC. Doped vanadium oxide for optical switching films. Sol Energy Mater 14, 205-214 (1986).

33. Wang XJ, Liu YY, Li DH, Feng BH, He ZW et al. Structural and optical properties of tungsten-doped vanadium dioxide films. Chin Phys B 22, 066803 (2013).

34. Zhang YF, Zhang JC, Zhang XZ, Huang C, Zhong YL et al. The additives $\mathrm{W}, \mathrm{Mo}, \mathrm{Sn}$ and Fe for promoting the formation of $\mathrm{VO}_{2}(\mathrm{M})$ and its optical switching properties. Mater Lett 92, 61-64 (2013).

35. Liu SJ, Fang HW, Su YT, Hsieh JH. Metal-insulator transition characteristics of Mo- and Mn-doped $\mathrm{VO}_{2}$ films fabricated by magnetron cosputtering technique. Jpn J Appl Phys 53, 063201 (2014).

36. Khan GR, Asokan K, Ahmad B. Room temperature tunability of Mo-doped $\mathrm{VO}_{2}$ nanofilms across semiconductor to metal phase transition. Thin Solid Films 625, 155-162 (2017).

37. Rajeswaran $B$, Umarji AM. Effect of $W$ addition on the electrical switching of $\mathrm{VO}_{2}$ thin films. AIP Adv 6, 035215 (2016).

38. Muraoka $\mathrm{Y}$, Hiroi Z. Metal-insulator transition of $\mathrm{VO}_{2}$ thin films grown on $\mathrm{TiO}_{2}(001)$ and (110) substrates. Appl Phys Lett 80, 583-585 (2002).

39. Fan LL, Chen S, Luo ZL, Liu QH, Wu YF et al. Strain dynamics of ultrathin $\mathrm{VO}_{2}$ film grown on $\mathrm{TiO}_{2}(001)$ and the associated phase transition modulation. Nano Lett 14, 4036-4043 (2014).

40. Ono M, Chen KF, Li W, Fan SH. Self-adaptive radiative cooling based on phase change materials. Opt Express 26, A777-A787 (2018).

41. Kort-Kamp WJM, Kramadhati S, Azad AK, Reiten MT, Dalvit
DAR. Passive radiative "thermostat" enabled by phase-change photonic nanostructures. ACS Photonics 5, 4554-4560 (2018).

42. Hecht E. Optics (Pearson Higher Education, Harlow, 2017).

43. Yeh P. Optical Waves in Layered Media 95 (Wiley Online Library, New York, 1988).

44. Kirchhoff G. Ueber das Verhältniss zwischen dem Emissionsvermögen und dem Absorptionsvermögen der Körper für Wärme und Licht. Ann Phys 185, 275-301 (1860).

45. Berk A, Anderson GP, Acharya PK, Bernstein LS, Muratov L et al. MODTRAN5: 2006 update. Proc SPIE 6233, 62331F (2006).

46. Brandrup J, Immergut EH. Polymer Handbook 2nd ed (John Wiley and Sons, London, 1975).

47. Wypych G. PMMA polymethylmethacrylate. Handbook of Polymers 2nd ed 450-454 (Elsevier, Oxford, 2012).

48. Haynes WM, Lide DR, Bruno TJ. CRC Handbook of Chemistry and Physics (CRC Press, Boca Raton, 2014).

49. Dargys A, Kundrotas J. Handbook on Physical Properties of $\mathrm{Ge}, \mathrm{Si}, \mathrm{GaAs}$ and InP (Science and Encyclopedia Publ., Vilniys, 1994).

50. Tipler PA, Mosca G. Physics for Scientists and Engineers 6th ed (WH Freeman, 2007).

51. Grove AS. Physics and Technology of Semiconductor Devices (Wiley, New York, 1967).

52. National Center for Biotechnology Information. PubChem Database. Vanadium dioxide, $C I D=82849$. https://pubchem. ncbi.nlm.nih.gov/compound/Vanadium-dioxide.

53. Chase Jr MW. NIST-JANAF Thermochemical Tables 4th ed (American Chemical Society and American Institute of Physics, New York, 1998).

54. Korea Meteorological Administration (ASOS). https://data.kma. go.kr/resources/html/en/aowdp.html.

55. Shi Y, Li W, Raman A, Fan SH. Optimization of multilayer optical films with a memetic algorithm and mixed integer programming. ACS Photonics 5, 684-691 (2018).

56. Chae D, Kim M, Jung PH, Son S, Seo J et al. Spectrally selective inorganic-based multilayer emitter for daytime radiative cooling. ACS Appl Mater Interfaces 12, 8073-8081 (2020).

57. Naghshine BB, Saboonchi A. Optimized thin film coatings for passive radiative cooling applications. Opt Commun $\mathbf{4 1 0}$, 416-423 (2018)

\section{Acknowledgements}

This work was financially supported by the Green Science program funded by POSCO, and the National Research Foundation (NRF) grants (NRF2019R1A2C3003129, CAMM-2019M3A6B3030637, NRF-2019R1A5A8080 290, and NRF-2018M3D1A1058997) funded by the Ministry of Science and ICT, Republic of Korea. M. Kim acknowledges the Global Ph.D. fellowship (NRF-2017H1A2A1043204) from the Ministry of Education, Republic of Korea. D. Lee acknowledges the PIURI fellowship funded by POSTECH. Y. Yang acknowledges a fellowship from Hyundai Motor Chung Mong-Koo Foundation.

\section{Author contributions}

M. K. Kim and D. S. Lee contributed equally to this work. J. S. Rho, M. K. Kim and D. S. Lee conceive the idea and initiate the project. M. K. Kim and D. S. Lee designed the system, performed numerical simulation and wrote manuscript. M. K. Kim and Y. H. Yang analyzed the data. All authors read and approved the final manuscript. J. S. Rho guided the entire work.

\section{Competing interests}

The authors declare no competing financial interests. 